\title{
Modelos de elevação para obtenção de atributos topográficos utilizados em mapeamento digital de solos
}

\author{
Helena Saraiva Koenow Pinheiro(1), César da Silva Chagas ${ }^{(2)}$, Waldir de Carvalho Júnior(2) \\ e Lúcia Helena Cunha dos Anjos ${ }^{(1)}$
}

\begin{abstract}
(1)Universidade Federal Rural do Rio de Janeiro, Departamento de Solos, BR-465, Km 47, CEP 23890-000 Seropédica, RJ. E-mail: lenask@gmail.com, lanjos@ufrrj.br (2)Embrapa Solos, Rua Jardim Botânico, № 1.024, Jardim Botânico, CEP 22460-000 Rio de Janeiro, RJ. E-mail: chagas.rj@gmail.com, waldircj@cnps.embrapa.br
\end{abstract}

Resumo - O objetivo deste trabalho foi avaliar modelos digitais de elevação (MDE), obtidos por diferentes fontes de dados, e selecionar um deles para derivar variáveis morfométricas utilizadas em mapeamento digital de solos. O trabalho foi realizado na Bacia Guapi-Macacu, RJ. Os dados primários utilizados nos modelos gerados por interpolação (MDE-carta e MDE-híbrido) foram: curvas de nível, drenagem, pontos cotados e dados de sensor remoto transformados em pontos. Utilizaram-se, na comparação, modelos obtidos por sensor remoto e por aerorrestituição (MDE SRTM e MDE IBGE). Todos os modelos apresentaram resolução espacial de $30 \mathrm{~m}$. A avaliação dos modelos de elevação foi baseada na análise de: atributos derivados (declividade, aspecto e curvatura); depressões espúrias; comparação entre feições derivadas a partir dos modelos e as originais, oriundas de cartas planialtimétricas; e análise das bacias de contribuição derivadas. O modelo digital de elevação híbrido apresenta qualidade superior à dos demais modelos.

Termos para indexação: atributos de terreno, bacias de contribuição, levantamento de solo, modelagem digital, SRTM.

\section{Elevation models for obtaining terrain attributes used in digital soil mapping}

\begin{abstract}
The objective of this work was to evaluate digital elevation models (DEM) obtained by different data sources and to select one of them for deriving morphometric variables used in digital soil mapping. The work was performed in the Guapi-Macacu river basin, RJ, Brazil. The primary data used in the models generated by interpolation (DEM map and DEM hybrid) were: contour lines, drainage, elevation points, and remote sensor data transformed into points. The obtained models by remote sensing and aero-restitution (DEM SRTM and DEM IBGE) were used in the comparison. All models showed spatial resolution of $30 \mathrm{~m}$. The elevation model evaluations were based on: the terrain derived attribute analysis (slope, aspect, and curvature); spurious depressions (sink); comparison between features derived from the models and the original ones originated from planialtimetric maps; and the analysis of derived watersheds. The DEM hybrid showed a superior quality than the other models.
\end{abstract}

Index terms: terrain attribute, watersheds, soil survey, digital modelling, SRTM.

\section{Introdução}

$\mathrm{O}$ uso de técnicas computacionais modernas para manipulação, análise e distribuição de dados espaciais tem contribuído para maior rapidez e menor custo de execução dos levantamentos de solos no mundo. Essas técnicas tornam o mapeamento mais quantitativo e possibilitam avaliar as incertezas. Dados de sensoriamento remoto orbital e atributos do terreno, derivados de modelos digitais de elevação (MDE), vêm sendo usados para compreensão das relações espaciais e temporais entre classes de solos e diferentes variáveis ambientais (Dobos et al., 2000; McBratney et al., 2003; Minasny \& McBratney, 2007; Sanchez et al., 2009).

Os atributos topográficos, também designados como variáveis morfométricas (Shary et al., 2002), são geralmente derivados do MDE por meio de métodos de análise digital do terreno. Estes atributos são comumente utilizados como variáveis auxiliares na predição espacial dos padrões solo-paisagem e contribuem para o aperfeiçoamento do mapeamento de classes e de propriedades do solo, como espessura de horizontes, elementos na solução do solo, textura, cor, umidade, entre outras (Gessler et al., 2000). 
É crescente a demanda por modelos de elevação e atributos morfométricos derivados, para representação contínua do terreno em formato digital, largamente usada em análise da paisagem e em outras aplicações. No entanto, pouca atenção tem sido empregada na padronização dos procedimentos para obtenção e avaliação da qualidade, na representação das variáveis morfométricas derivadas (Oksanen \& Sarjakoski, 2005; Lin \& Oguchi, 2006).

Os MDE podem ser obtidos por várias técnicas (Hutchinson \& Gallant, 2000), pela interpolação de feições vetoriais (pontos cotados, curvas de nível e hidrografia) ou diretamente de sensores remotos. Os MDE obtidos por sensoriamento remoto orbital estão disponíveis mundialmente, com grande cobertura de área por cena e baixo custo de processamento; porém, a qualidade das informações depende da rugosidade $\mathrm{e}$ da declividade do terreno.

A acurácia do MDE e dos atributos topográficos derivados depende da qualidade e densidade dos dados primários, da estrutura dos dados (matricial, vetorial), da resolução espacial, da complexidade topográfica da área e dos algoritmos utilizados na interpolação (Thompson et al., 2001). Modelos digitais oriundos do Shuttle Radar Topography Mission (SRTM) podem apresentar erros, atribuídos a ruídos na aquisição e transferência dos dados, e à geometria da órbita. Além disso, os MDE também podem ser influenciados pelas características do terreno e comumente apresentam qualidade superior em áreas de relevo pouco movimentado e declive suave (Jarvis et al., 2004).

Há várias maneiras de se avaliar a qualidade de um MDE (Thompson et al., 2001). O método tradicional confronta uma amostra de pontos de elevação do MDE contra pontos de elevação provenientes de fonte de dados de maior acurácia (valores considerados reais). O grau de concordância entre estes modelos é referido como a raiz quadrada do erro médio quadrático da elevação (RMSE). Uma vez que as aplicações do MDE dependem de representações da forma da superfície e estrutura da drenagem, medidas absolutas dos erros de elevação não fornecem uma avaliação completa da qualidade de um MDE (Wise, 2000). Hutchinson \& Gallant (2000) propõem medidas não clássicas de análise - uso de pontos de referência sem elevada exatidão - como critérios para avaliação de MDE, tais como: comparação visual entre feições vetoriais derivadas e originais, e análise das bacias de contribuição.

A avaliação da qualidade de modelos de elevação é importante no mapeamento digital de solos, em razão da relação direta entre a variabilidade dos atributos do terreno e a pedogênese condicionada pelo fluxo das águas, temperatura, vegetação e remoção e deposição de material. Vários estudos mostram que o uso de atributos de terreno derivados do MDE é eficiente na predição de propriedades e classes do solo. (Gessler et al., 2000; Hansen et al., 2009; Chagas et al., 2010a, 2010b; Carvalho Júnior et al., 2011). Em razão da importância do MDE como fonte primordial de dados espaciais para a caracterização das diferentes formas da paisagem, é importante uma análise detalhada da qualidade do MDE e de alguns atributos derivados comumente utilizados em mapeamento digital de solos.

O objetivo deste trabalho foi avaliar modelos digitais de elevação (MDE), obtidos por diferentes fontes de dados, e selecionar um deles para derivar variáveis morfométricas utilizadas em mapeamento digital de solos.

\section{Material e Métodos}

A Bacia Hidrográfica do Rio Guapi-Macacu, localizada entre as coordenadas UTM 7.488.481 e $7.526 .005 \mathrm{~m} \mathrm{~S}$ e 699.292 e $752.193 \mathrm{~m} \mathrm{~W}$, tem área de contribuição de $1.250,78 \mathrm{~km}^{2}$, que abrange o Município de Cachoeiras de Macacu e partes de Itaboraí e de Guapimirim, RJ. O clima da região, conforme classificação de Köppen, é do tipo Aw (tropical chuvoso, com inverno frio e seco). A temperatura média anual é de $23^{\circ} \mathrm{C}$, e a precipitação média anual supera $1.200 \mathrm{~mm}$ (Hwa \& Hora, 2010).

Os MDE foram obtidos de quatro formas distintas: MDE-carta, a partir da interpolação de curvas de nível com equidistância de $20 \mathrm{~m}$, da hidrografia e de pontos cotados extraídos de cartas planialtimétricas do IBGE (folhas Itaboraí - SF-23-Z-B-V-1, Itaipava - SF-23-Z-B-I-4, Nova Friburgo - SF-23-Z-B-II-4, Petrópolis - SF-23-Z-B-IV-2, Teresópolis SF-23-Z-B-II-3) e do DSG (folha Rio Bonito SF-23-Z-B-V-2), todas na escala de 1:50.000; MDE híbrido, por interpolação com as fontes de dados do MDE-carta +2.719 pontos de elevação extraídos do SRTM, disponível em http://www.dsr.inpe.br/topodata, 
para áreas da bacia com cotas inferiores a $20 \mathrm{~m}$, mais 5.672 pontos cotados extraídos de cartas planialtimétricas $(1: 10.000)$ de algumas áreas da bacia (Fundação Centro de Informações e Dados do Rio de Janeiro, 1996); MDE SRTM, dados do "Topodata", com 30 m de resolução espacial; e MDE IBGE, por processamento fotogramétrico analítico (Instituto Brasileiro de Geografia e Estatística, 2008), originalmente disponível em resolução espacial de $20 \mathrm{~m}$ e reamostrado para $30 \mathrm{~m}$.

Inicialmente, os dados foram pré-processados para possibilitar a geração e utilização dos MDE, o que envolveu: a união das cartas topográficas para as feições vetoriais de curvas de nível, hidrografia e pontos cotados; a definição da projeção cartográfica para Universal Transversa de Mercator (UTM), datum horizontal Córrego Alegre Zona 23S; e a checagem das informações dos pontos cotados, curvas de nível e drenagem, tendo-se eliminado os erros de posicionamento, valores, sentido e continuidade.

A localização da bacia hidrográfica e dos pontos cotados, usados como dados primários para elaborar os modelos gerados por interpolação, estão na Figura 1 . Os pontos cotados extraídos do SRTM foram obtidos por conversão do arquivo matricial, com $30 \mathrm{~m}$ de resolução, para arquivo vetorial de pontos, com posterior seleção dos pontos com elevação abaixo da curva de nível de $20 \mathrm{~m}$. Os procedimentos foram efetuados no ArcGIS Desktop v.10 (Environmental Systems Research Institute, 2010). Este artifício foi aplicado para suprir a carência de dados de elevação (curvas de nível ou pontos de elevação) nas áreas mais baixas da bacia, próximas à Baía da Guanabara, que poderiam interferir no processo de interpolação, conforme destacado por Wise (2000).

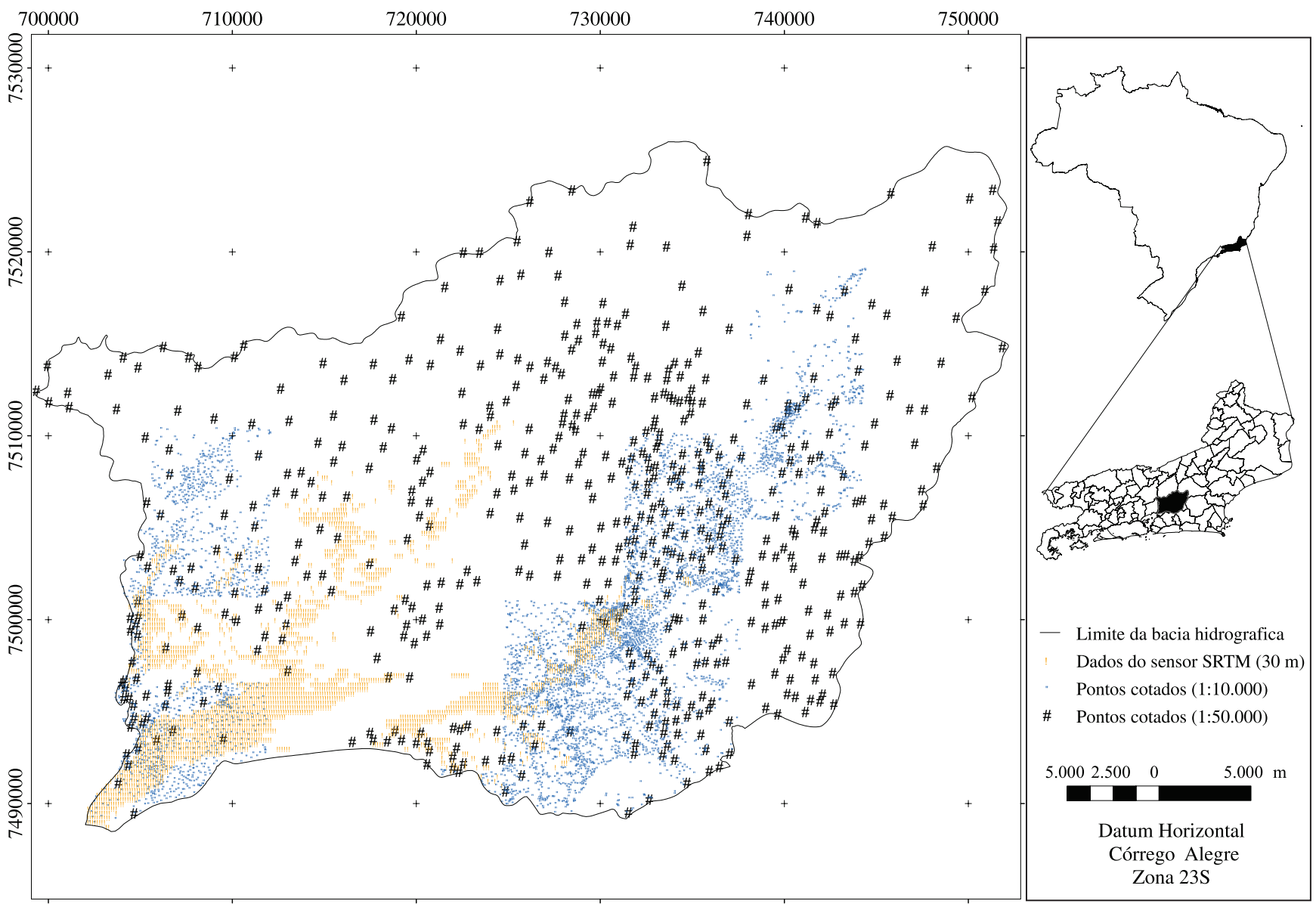

Figura 1. Localização dos dados primários de elevação dos pontos cotados e da bacia hidrográfica do rio Guapi-Macacu, no Estado do Rio de Janeiro, Brasil. 
Os MDE-carta e híbrido foram gerados com a ferramenta Topo to Raster do ArcGIS Desktop v.10, que implementa um método de interpolação específico para a criação de modelo digital de elevação hidrologicamente consistente, a partir da combinação de métodos de interpolação local e global, com base no programa Anudem desenvolvido por Hutchinson (1993).

Os dados do SRTM estão disponíveis no Instituto Nacional de Pesquisas Espaciais (Inpe), para todo o território brasileiro, com resolução espacial de $30 \mathrm{~m}$. Vale destacar, que os dados do Topodata passaram por um processo de refinamento para aumento da resolução espacial original de 3 arcossegundos ( $\sim 90 \mathrm{~m})$ para 1 arcossegundo $(\sim 30 \mathrm{~m})$, por meio de krigagem (Valeriano, 2004). Após a obtenção dos dados, foram unidas as diferentes cenas, com posterior adequação do sistema de projeção e seleção da área correspondente à bacia hidrográfica em estudo. Este modelo foi denominado MDE SRTM.

O MDE IBGE é um produto do projeto RJ-25, do mapeamento sistemático brasileiro realizado pelo Instituto Brasileiro de Geografia e Estatística (2008). Os MDE são derivados de processamento fotogramétrico analítico, a partir de aerolevantamento (escala aproximada de 1:30.000 e resolução de 0,7 m) e algoritmos de extração altimétrica por correlação de imagens (aerorrestituição espacial), com exatidão variável em consequência das características da região (Instituto Brasileiro de Geografia e Estatística, 2008). Os dados originais com resolução espacial de $20 \mathrm{~m}$ foram reamostrados para $30 \mathrm{~m}$, com uso do algoritmo de reamostragem por atribuição dos vizinhos mais próximos ("nearest neighbor") no ArcGIS Desktop v.10, o que possibilitou a comparação com os demais MDE. A resolução espacial de $30 \mathrm{~m}$ foi escolhida em razão da compatibilidade com outras variáveis ambientais, como por exemplo imagens do sensor TM do LANDSAT 5.

Os MDE foram avaliados qualitativamente, com base na representação dos aspectos da superfície, conforme Hutchinson \& Gallant (2000) e Wise (2000). Os autores propõem como critérios: a análise do número e extensão das depressões espúrias; a comparação entre as curvas de nível derivadas e as curvas de nível originais; a comparação entre a drenagem mapeada e a drenagem numérica derivada pelos diferentes MDE; e a análise das bacias de contribuição derivadas. As depressões espúrias - células com valores incoerentes - foram removidas por processo repetitivo que assume valores concordantes com a hidrografia (Dobos et al., 2000).

A derivação da rede de drenagem é sensivelmente afetada pela presença de depressões espúrias em um MDE que podem comprometer as análises futuras do mapeamento digital de solos (Wise, 2000). Assim, a avaliação contou, em primeira instância, com a determinação do número e extensão das depressões de cada um dos quatro MDE, sem a utilização de processos de filtragem ou suavização ("smoothing"). As demais avaliações foram realizadas nos MDE após a eliminação das depressões espúrias. Este procedimento foi simplificado pelo comando 'spatial analyst tools/ hidrology/sink', no programa ArcGIS Desktop v.10.

A derivação das curvas de nível dos MDE foi executada no módulo 'spatial analyst tools/surface/ contour' do ArcGIS Desktop v.10. No cálculo do escoamento acumulado, para a obtenção da rede de drenagem numérica, foi atribuído um valor numérico associado ao número de células que fluem para uma determinada célula. Assim, células com valores superiores a 500 foram identificadas como pertencentes à drenagem numérica e com aproximadamente $\mathrm{o}$ mesmo nível de detalhe da rede de drenagem mapeada (Chagas et al., 2010a). Por meio de operação de álgebra de mapas e posterior transformação do tema matricial para vetorial, foi possível realizar a comparação entre as drenagens numéricas derivadas e a original. Todos estes procedimentos foram realizados no ArcGIS Desktop v.10.

As bacias de contribuição derivadas foram obtidas pela função 'watershed' no ArcGIS Desktop v.10 e avaliadas em função de sua área e perímetro. A qualidade dos modelos que representam as formas do relevo é um fator de grande importância, uma vez que implica diretamente na qualidade dos produtos finais da modelagem espacial. Portanto, critérios de análise que consideram as implicações do MDE nos produtos derivados (atributos primários) foram utilizados para complementar a avaliação. Os atributos primários declividade, aspecto e curvatura - foram derivados no módulo 'spatial analyst tools/surface' do ArcGIS Desktop v.10.

Os critérios adotados tiveram por finalidade subsidiar a escolha de um MDE adequado, para a derivação de atributos do terreno e interpretações das relações solo-paisagem na bacia hidrográfica avaliada. 


\section{Resultados e Discussão}

A avaliação dos modelos com base na análise dos atributos primários derivados torna-se mais compreensível quando estes são reclassificados em intervalos de classes (Figura 2), o que permite identificar as principais diferenças entre os modelos e as possíveis implicações na representação do relevo. Os erros presentes no MDE podem confundir as relações esperadas entre atributos do terreno e as condições locais da paisagem e podem ser amplificados quando atributos do terreno são derivados do modelo digital de elevação (Chagas et al., 2010a). A precisão de MDE derivados de dados hidrológicos está diretamente relacionada à resolução vertical e rugosidade do terreno. Nas áreas de declives suaves, os parâmetros hidrológicos geralmente são mais confiáveis (Hengl et al., 2004).

Com relação à altimetria, as principais diferenças entre os modelos ocorreram nas áreas com cotas inferiores a $50 \mathrm{~m}$ (Figura $2 \mathrm{~A}$ ), que representam cerca de $40 \%$ da área da bacia, em sua maior parte de superfícies planas. Chagas et al. (2010a) encontrou as maiores discordâncias entre os MDE SRTM e carta nas partes mais baixas e planas da paisagem. Neste caso, o MDE IBGE tende a subestimar as áreas com elevação inferior a $20 \mathrm{~m}$ e a superestimar as áreas entre $20 \mathrm{e}$ $50 \mathrm{~m}$. O mesmo comportamento foi verificado para o MDE-carta, porém de forma menos expressiva.

A avaliação da declividade mostrou relação direta com a altimetria, em que as maiores diferenças foram observadas para as classes de declive inferiores a $20 \%$, associadas às áreas com elevações inferiores a $50 \mathrm{~m}$ (Figura 2 B). O MDE-carta estimou a menor área para a classe de declive inferior a $3 \%$, o que corrobora a expectativa de carência de dados ou erros relativos às áreas de planícies da bacia hidrográfica.

A derivação de atributos a partir de modelos inconsistentes pode gerar informações truncadas, relacionadas principalmente a fenômenos hidrológicos e de transporte de material, como a ocorrência de áreas alagadas, e zonas de oxidação e redução, remoção e deposição. Dessa forma, esse truncamento pode prejudicar o reconhecimento dos diferentes tipos de solos e resultar em interpretações errôneas. Embora os critérios adotados para a avaliação dos MDE sejam referentes ao comportamento hidrológico, o desenvolvimento do solo depende diretamente do movimento da água pela paisagem e, em razão desta interdependência, podem ser aplicados para a seleção de modelos de elevação e atributos derivados para uso em mapeamento digital de solos.

De modo geral, os modelos apresentaram semelhanças na derivação das classes de curvatura (Figura 2 C). Entretanto, a divisão em classes possibilitou detectar diferenças no MDE IBGE, em compa-
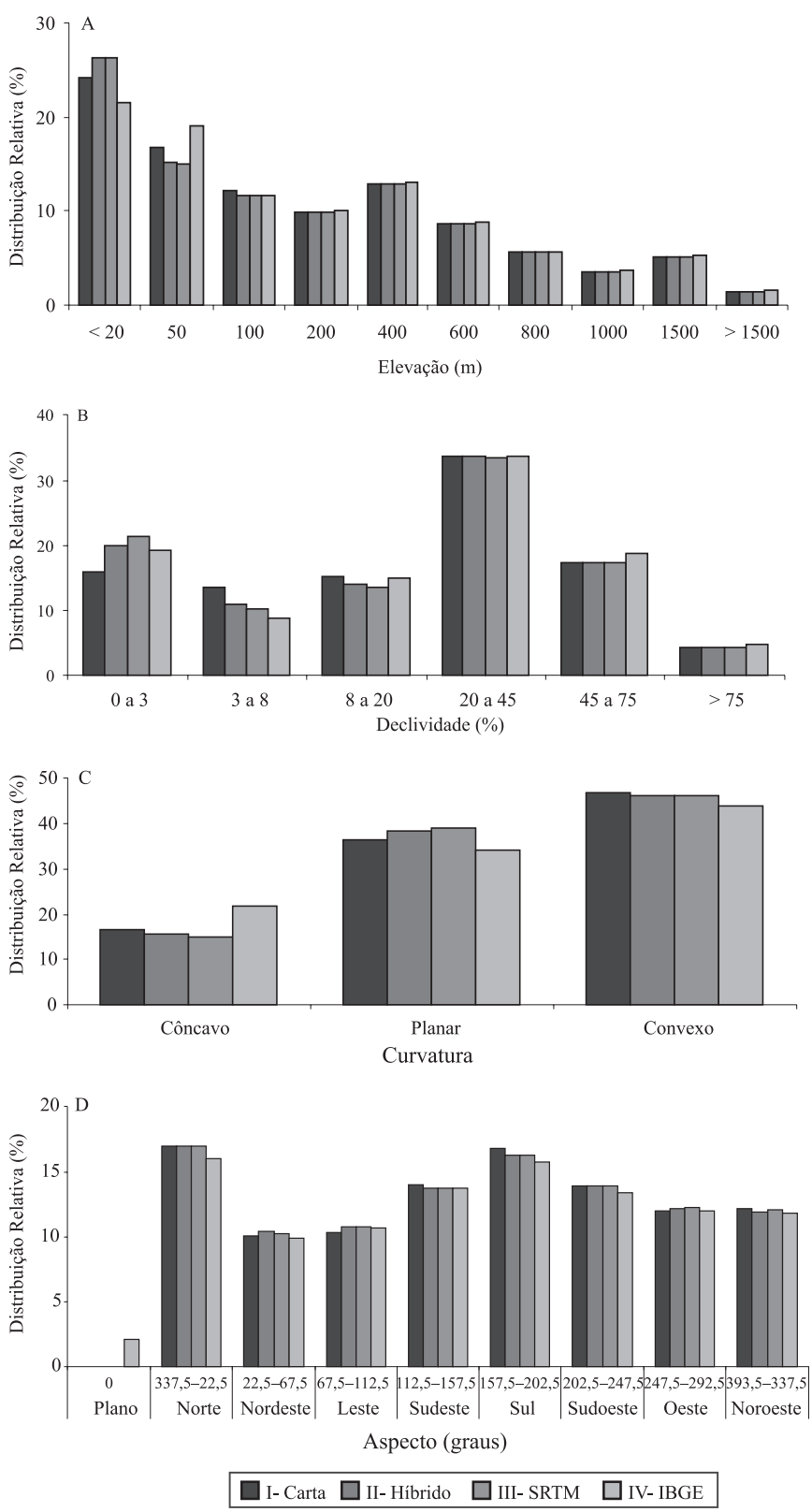

Figura 2. Distribuição relativa da classificação dos atributos primários, para os quatro modelos avaliados: A, elevação (m); B, declividade (\%); C, curvatura (adimensional); D, aspecto (graus). 
ração aos demais modelos, uma vez que o número de células da classe de curvatura côncava foi superestimado e, na classe de curvatura planar, subestimado. As diferenças detectadas para esses atributos entre os modelos são importantes, pois afetam diretamente a predição e delimitação dos solos de várzea, como os Planossolos, Gleissolos e Neossolos Flúvicos.

Apesar da semelhança entre os modelos quanto ao aspecto, que evidencia a dominância das encostas com orientação sul, sudeste e sudoeste, a divisão em classes mostrou que o MDE IBGE (Figura 2 D) identificou mais áreas como planas, ou seja, sem faces orientadas para nenhum lado, do que os demais modelos, diferentemente da realidade do terreno.

Foram necessárias de quatro a seis repetições da sequência de operações para a remoção completa das depressões espúrias. A avaliação da qualidade dos modelos, no que tange ao número e extensão de depressões espúrias, destaca a diferença entre as formas de aquisição dos modelos, em comparação àqueles obtidos de medidas diretas (MDE SRTM e MDE IBGE) com os de indiretas (MDE-carta e MDE-híbrido) (Tabela 1). Observou-se que os modelos obtidos por medidas indiretas têm menor número e extensão de células de depressões espúrias, em comparação ao MDE SRTM e MDE IBGE. Houve também diferença marcante no número e extensão das depressões espúrias, entre os modelos obtidos por interpolação dos modelos oriundos de sensores remotos e aerorrestituição.

Observou-se ligeiro aumento no número de depressões espúrias do MDE-carta para o MDE-híbrido, porém, em relação à área total, em ambos, essas células não ultrapassaram $0,3 \%$. Os MDE SRTM e IBGE apresentaram valores maiores para o número de depressões espúrias, com valores cerca de três vezes maiores que os obtidos pelos MDE-carta e híbrido, o que destaca os efeitos da forma de obtenção de dados.

Tabela 1. Número de depressões espúrias e de células envolvidas, e relação com a área total da Bacia Hidrográfica Guapi-Macacu (RJ), para os quatro modelos digitais de elevação (MDE) avaliados.

\begin{tabular}{lccc}
\hline MDE & $\begin{array}{c}\text { Número de } \\
\text { depressões }\end{array}$ & $\begin{array}{c}\text { Número de células } \\
\text { envolvidas }\end{array}$ & $\begin{array}{c}\text { Relação com a área total } \\
(\%)\end{array}$ \\
\hline Carta & 1.519 & 3.037 & 0,21 \\
Híbrido & 1.731 & 3.472 & 0,25 \\
SRTM & 6.826 & 14.591 & 1,05 \\
IBGE & 5.089 & 26.233 & 1,88 \\
\hline
\end{tabular}

SRTM, Shuttle Radar Topography Mission.
Foi possível observar que, entre os MDE SRTM e IBGE, houve uma relação inversa entre o número de células de depressões e a extensão em que estas se agruparam. Hengl et al. (2004) destacaram que o elevado número de depressões nos modelos derivados de sensores remotos pode ser atribuído a erros intrínsecos ao método de coleta de dados pelos sensores.

A avaliação de modelos de elevação é importante no mapeamento digital de solos, em razão da relação direta entre a variabilidade dos atributos do terreno e a pedogênese condicionada pelo fluxo das águas, temperatura, vegetação e remoção e deposição de material. Visto que as aplicações dos MDE dependem da representação da forma da superfície e estrutura de drenagem, valores absolutos de erros não fornecem avaliação completa da qualidade do MDE, por isso é necessário complementar a análise com critérios qualitativos (Wise, 2000). Hutchinson \& Gallant (2000) destacam que a comparação das curvas de nível derivadas fornece uma avaliação sensitiva sobre a estrutura do terreno e é de grande utilidade quanto à sensibilidade aos erros de elevação na fonte de dados. A comparação visual entre curvas de nível derivadas dos MDE e originais das cartas planialtimétricas foi parte dessa avaliação complementar (Figura 3).

A melhor concordância entre as curvas de nível derivadas e as originais foi observada nos MDE-carta e híbrido. Os topos de morros apresentaram pouca variação em relação às linhas originais; porém, na baixada, o MDE-carta apresentou feições incompatíveis com a base original de dados. Os MDE SRTM e IBGE mostraram maior incoerência e deslocamento, na comparação com as feições originais. No caso do processamento analítico, artefatos - como áreas de sombra, por exemplo - foram criados em razão do processo de extração e de "ruídos" na obtenção pelos sensores remotos. Estas anomalias foram representadas por desníveis ou descontinuidades bem como por tabuleiros de forma triangular ou quadrangular, e representaram padrões incompatíveis com feições naturais do relevo. Observações similares foram relatadas por Chagas et al. (2010a).

A comparação visual entre a drenagem numérica derivada e a drenagem original da carta topográfica mostrou os efeitos das diferentes formas de obtenção dos modelos (Figura 4). Foram observadas, além de falhas, a criação de feições inexistentes, deslocamentos e retificação de cursos d'água, principalmente nos 
modelos obtidos por medidas diretas. Os dados do SRTM não são previamente editados e podem conter erros provenientes de "ruídos" e falhas, principalmente em áreas íngremes (Chagas et al., 2010a). As feições derivadas dos modelos gerados por interpolação apresentaram maior concordância com a base de dados original, em que se destacou o MDE-híbrido, que mostrou qualidade superior à dos demais modelos.

De forma geral, foi possível verificar melhor concordância das feições derivadas oriundas dos MDE obtidos por interpolação (carta e híbrido). Os demais modelos (SRTM e IBGE) tiveram baixa concordância, o que evidenciou, nestes casos, a presença de erros na fonte de dados. Embora se tenha utilizado os dados de curvas de nível e hidrografia, na confecção dos MDEs gerados por interpolação, os resultados alcançados mostraram diferenças entre os dois modelos.
O MDE-carta apresentou resultados não satisfatórios, com pior concordância entre as feições, principalmente no que tange à comparação da rede de drenagem derivada e a rede mapeada. Na comparação de feições derivadas e originais, o fato de se utilizar os mesmos dados primários utilizados na interpolação nem sempre implica melhor concordância das feições derivadas dos modelos (Saunders, 1999).

A análise das bacias de contribuição derivadas permitiu detectar diferenças sutis, porém relevantes, na qualidade dos modelos, o que corrobora as observações de Wise (2000). Os modelos interpolados (MDE-carta e híbrido) mostraram maior sensibilidade às direções de fluxo e maior aproximação com a área total da bacia hidrográfica. Algumas das feições incoerentes dos MDE SRTM e IBGE podem estar relacionadas às etapas do pós-processamento dos dados disponibilizados,
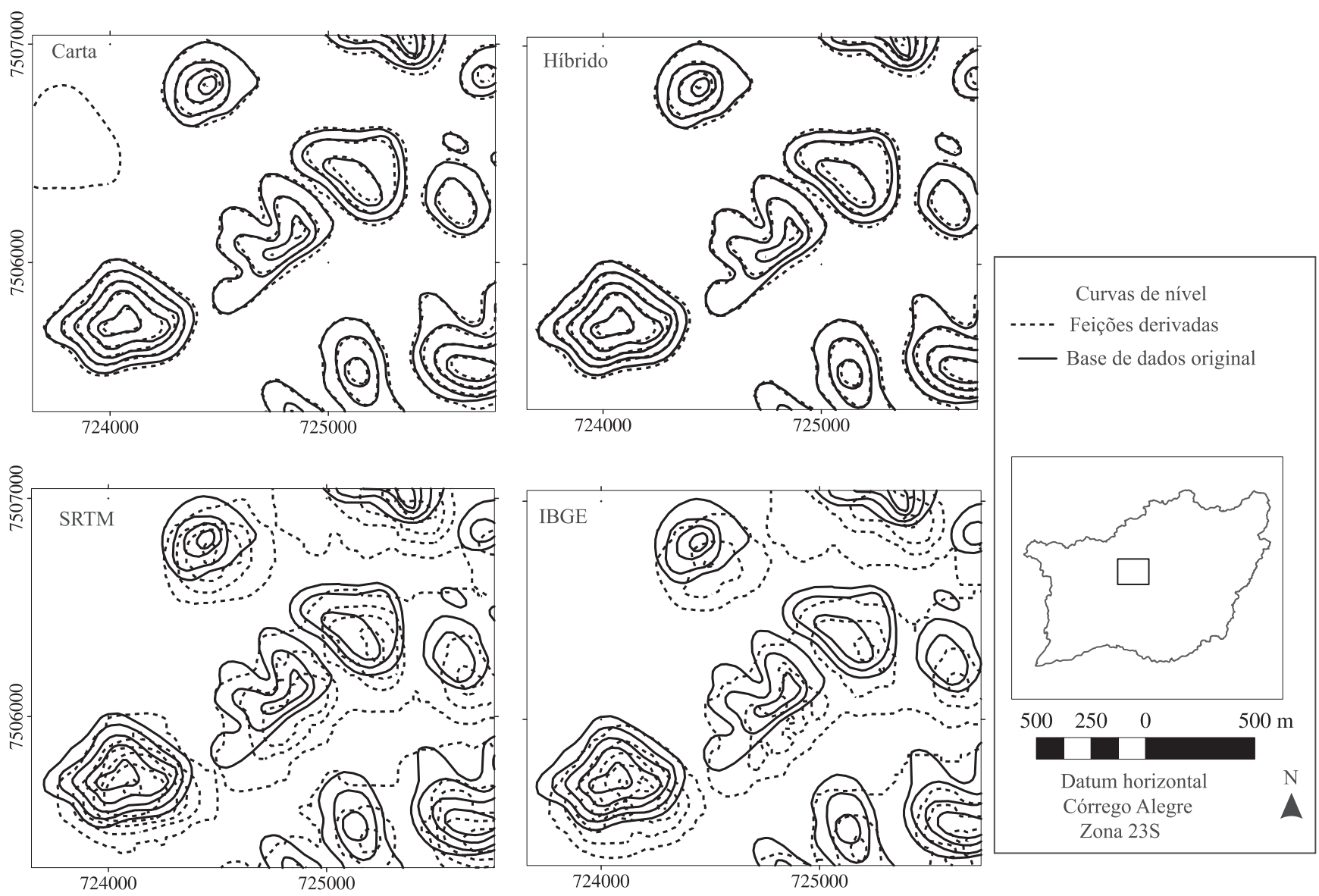

Figura 3. Visualização comparativa entre curvas de nível originais e derivadas dos modelos digitais de elevação (MDE), para os modelos carta, híbrido, SRTM e IBGE avaliados. 
como o corte e mosaico das imagens. Os MDE-carta e híbrido apresentaram padrões similares; porém, no MDE-carta, foram criadas feições mais destoantes dos limites, principalmente nas partes mais rebaixadas, próximas ao exutório da bacia hidrográfica.

Todos os grupos de dados reconheceram as três grandes sub-bacias que correspondem aos principais cursos d'água e zonas hidrológicas (rios Guapimirim e Macacu e foz na Baía de Guanabara), e, de forma geral, não apresentaram grandes diferenças visuais entre os modelos, senão as citadas anteriormente. $\mathrm{Na}$ avaliação das bacias de contribuição, foram comparados a área e o perímetro da bacia derivada de cada modelo com a feição vetorial que delimita a área de estudo (Figura 5). Em todos os modelos, a área de contribuição da bacia derivada foi menor do que a do limite pré-determinado, e o inverso foi observado quanto aos perímetros (Tabela 2). Este fato é explicado pela criação de artefatos nos limites da bacia, representados por reentrâncias irregulares, o que explica o aumento do perímetro decorrente do processo de derivação automatizada dessas bacias. O MDE SRTM, entre os modelos testados, foi o que mostrou as maiores diferenças com relação ao limite original para área e perímetro da bacia. O MDE IBGE apresentou o mesmo padrão; porém, os valores relativos para diferença foram inferiores ao anterior. Os demais modelos (MDE-carta e híbrido) produziram valores semelhantes, embora com relação inversa entre área e perímetro, o que pode indicar deformações, como reentrâncias nas proximidades do limite da bacia hidrográfica.

Constatou-se que as distintas formas de obtenção dos MDE proporcionaram que estes apresentassem diferenças entre nos atributos derivados, o que destacou a importância das interpretações das relações solo-paisagem. Em geral, os resultados da avaliação
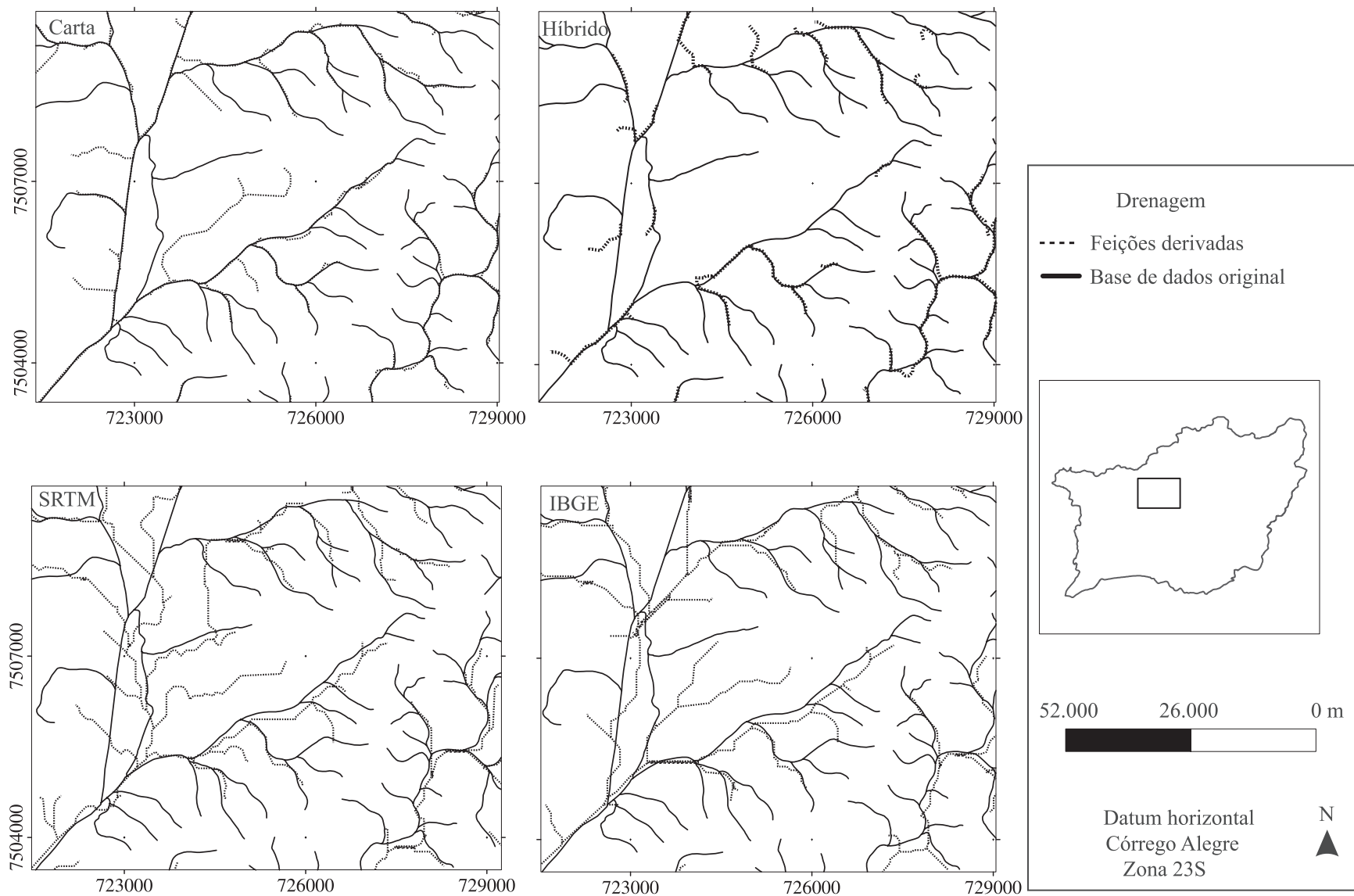

Figura 4. Comparação entre drenagem da base de dados derivada e original dos modelos digitais de elevação (MDE), para os modelos carta, híbrido, SRTM e IBGE avaliados. 

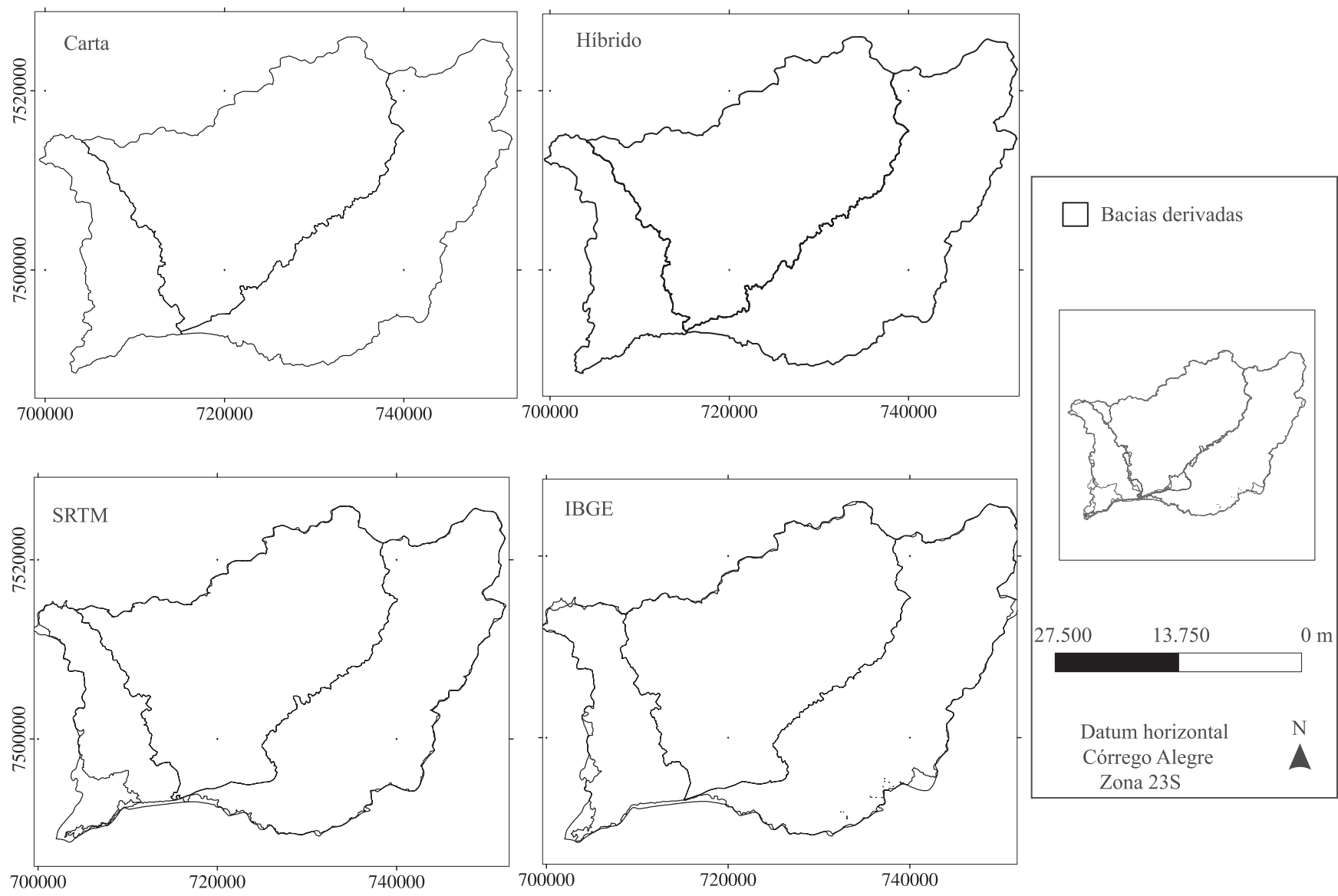

Córrego Alegre

Zona $23 \mathrm{~S}$

Figura 5. Bacias de contribuição derivadas dos modelos digitais de elevação (MDE), para os modelos carta, híbrido, SRTM e IBGE avaliados.

Tabela 2. Área e perímetro das bacias derivadas dos diferentes modelos gerados.

\begin{tabular}{lcccc}
\hline Modelos & $\begin{array}{c}\text { Área } \\
\text { (ha) }\end{array}$ & $\begin{array}{c}\text { Diferença relativa } \\
(\%)\end{array}$ & $\begin{array}{c}\text { Perímetro } \\
(\mathrm{m})\end{array}$ & $\begin{array}{c}\text { Diferença relativa } \\
(\%)\end{array}$ \\
\hline Carta & $123.977,93$ & 0,79 & $198.229,66$ & 3,44 \\
Híbrido & $124.250,61$ & 0,58 & $199.416,18$ & 4,06 \\
SRTM & $120.019,73$ & 3,96 & $223.566,59$ & 16,67 \\
IBGE & $121.793,51$ & 2,54 & $213.872,97$ & 11,61 \\
Total Bacia & $124.972,01$ & & $191.622,00$ & \\
\hline
\end{tabular}

SRTM, Shuttle Radar Topography Mission.

dos MDE para a Bacia Guapi-Macacu corroboram os de Chagas et al. (2010a), que observaram qualidade superior em modelos por interpolação de dados primários, em comparação aos obtidos por sensores remotos.

\section{Conclusões}

1. O número e extensão de depressões espúrias mostram diferenças entre os modelos derivados de curvas de nível e os modelos obtidos por sensor remoto e aerorrestituição fotogramétrica.

2. Os MDE-carta e IBGE apresentam qualidade inferior na avaliação com os atributos primários do terreno; em contrapartida, o MDE-híbrido e o MDE SRTM promovem melhor representação dos atributos da superfície do terreno, principalmente em áreas de planícies aluviais, com reduzida amplitude topográfica.

3. A comparação entre feições originais e derivadas dos MDE permite detectar diferenças entre os modelos e indica maior compatibilidade entre as feições derivadas dos MDE oriundos de interpolação de dados primários de elevação (MDE-carta e híbrido). 
4. A adição de pontos cotados extras ao MDE-carta confere maior qualidade final ao MDE-híbrido.

5. O MDE-híbrido é o modelo mais adequado para representar a superfície e para derivar os atributos topográficos empregados nas etapas posteriores do mapeamento digital de solos da Bacia Hidrográfica Guapi-Macacu.

\section{Agradecimentos}

Ao Curso de Pós-Graduação em Agronomia da Universidade Federal Rural do Rio de Janeiro, à Embrapa Solos, à Coordenação de Aperfeiçoamento de Pessoal de Nível Superior e à Fundação de Amparo à Pesquisa do Estado do Rio de Janeiro, pelo apoio técnico e financeiro e pela concessão bolsas.

\section{Referências}

CARVALHO JÚNIOR, W. de; CHAGAS, C. da S.; FERNANDES FILHO, E.I.; VIEIRA, C.A.O.; SCHAEFER, C.E.G.; BHERING, S.B.; FRANCELINO, M.R. Digital soilscape mapping of tropical hillslope areas by neural networks. Scientia Agricola, v.68, p.691-696, 2011.

CHAGAS, C. da S.; FERNANDES FILHO, E.; ROCHA, M.F. da; CARVALHO JÚNIOR, W. de; SOUZA NETO, N.C. de. Avaliação de modelos digitais de elevação para aplicação em um mapeamento digital de solos. Revista Brasileira de Engenharia Agrícola e Ambiental, v.14, p.218-226, 2010a.

CHAGAS, C. da S.; FERNANDES FILHO, E.I.; VIEIRA, C.A.O.; SCHAEFER, C.E.G.R.; CARVALHO JÚNIOR, W. de. Atributos topográficos e dados do Landsat7 no mapeamento digital de solos com uso de redes neurais. Pesquisa Agropecuária Brasileira, v.45, p.497-507, 2010 b.

DOBOS, E.; MICHELI, E.; BAUMGARDNER, M.F.; BIEHL, L.; HELT, T. Use of combined digital elevation model and satellite radiometric data for regional soil mapping. Geoderma, v.97, p.367-391, 2000.

ENVIRONMENTAL SYSTEMS RESEARCH INSTITUTE. Software: ArcGIS e ArcINFO. Version 10. Redlands: ESRI, 2010. FUNDAÇÃO CENTRO DE INFORMAÇÕES E DADOS DO RIO DE JANEIRO. Programa de despoluição da Baía de Guanabara - PDBG: folhas cartográficas. Rio de Janeiro: CIDE, 1996. Escala 1:10.000.

GESSLER, P.E.; CHADWICK, O.A.; CHAMRAN, F.; ALTHOUSE, L.; HOLMES, K. Modeling soil-landscape and ecosystem properties using terrain attributes. Soil Science Society of American Journal, v.64, p.2046-2056, 2000.

HANSEN, M.K.; BROWN, D.J.; DENNISON, P.E. Inductively mapping expert-derived soil-landscape units within dambo wetland catenae using multispectral and topographic data. Geoderma, v.150, p.72-84, 2009.
HENGL, T.; GRUBER S.; SHRESTHA, D.P. Reduction of errors in digital terrain parameters used in soil-landscape modelling. International Journal of Applied Earth Observation and Geoinformation, v.5, p.97-112, 2004.

HUTCHINSON, M.F. Development of continent-wide DEM with applications to terrain and climate analysis. In: GOODCHILD, M.F.; PARKS, B.O.; STEYAERT, L.T. (Ed.). Environmental modeling with GIS. New York: Oxford University, 1993. p.392-399.

HUTCHINSON, M.F.; GALLANT, J.C. Digital elevation models and representation of terrain shape. In: WILSON, J.P.; GALLANT, J.C. (Ed.). Terrain analysis: principles and applications. New York: John Wiley and Sons, 2000. p.29-50.HWA, C.S.; HORA, M. de A.G. da. (Coord.). Planejamento estratégico da região hidrográfica dos rios Guapi-Macacu e Caceribu-Macacu. Niterói: UFF/FEC, 2010. 544p.

INSTITUTO BRASILEIRO DE GEOGRAFIA E ESTATÍSTICA. Diretoria de Geociências. Departamento de Cartografia. Ponta do forno: folha SF-24-Y-A-IV-3-NE 2748-3-NE: modelo de elevação projeto RJ-25: metadados. Rio de Janeiro: IBGE, 2008. Disponível em: <ftp://geoftp.ibge.gov.br/>. Acesso em: 20 abr. 2011.JARVIS, A.; RUBIANO, J.; NELSON, A.; FARROW, A.; MULLIGAN, M. Practical use of SRTM data in the tropics - comparisons with digital elevation models generated from cartographic data. Cali: CIAT, 2004. 31p. (Working document, 198).

LIN, Z.; OGUCHI, T. DEM analysis on longitudinal and transverse profiles of steep mountainous watersheds. Geomorphology, v.78, p.77-89, 2006.

MCBRATNEY, A.B.; MENDONÇA-SANTOS, M.L.; MINASNY, B. On digital soil mapping. Geoderma, v.117, p.3-52, 2003.

MINASNY, B.; MCBRATNEY, A.B. Incorporating taxonomic distance into spatial prediction and digital mapping of soil classes. Geoderma, v.142, p.285-293, 2007.

OKSANEN, J.; SARJAKOSKI, T. Error propagation of DEM-based surface derivatives. Computer and Geoscience, v.31, p.1015-1027, 2005.

SANCHEZ, P.A.; AHAMED, S.; CARRÉ, F.; HARTEMINK, A.E.; HEMPEL, J.; HUISING, J.; LAGACHERIE, P.; MCBRATNEY, A.B.; MCKENZIE, N.J.; MENDONÇA-SANTOS, M. de L.; MINASNY, B.; MONTANARELLA, L.; OKOTH, P.; PALM, C.A.; SACHS, J.D.; SHEPHERD, K.D.; VÅGEN, T.-G.; VANLAUWE, B.; WALSH, M.G.; WINOWIECKI, L.A.; ZHANG, G.-L. Digital soil map of the world. Science, v.325, p.680-681, 2009.

SAUNDERS, W. Preparation of DEMs for use in environmental modeling analysis. In: ESRI INTERNATIONAL USER CONFERENCE, 1999, San Diego. Proceedings. San Diego: ESRI, 1999. p.26-30.

SHARY, P.; SHARAYA, L.; MITUSOV, A. Fundamental quantitative methods of land surface analysis. Geoderma, v.107, p.1-32, 2002.

THOMPSON, J.A.; BELL, J.C.; BUTLER, C.A. Digital elevation model resolution: effects on terrain attribute calculation and quantitative soil-landscape modelling. Geoderma, v.100, p.67-89, 2001. 
VALERIANO, M. de M. Modelo digital de elevação com dados SRTM disponíveis para a América do Sul. São José dos Campos: INPE, 2004. 72p.
WISE, S. Assessing the quality for hydrological applications of digital elevation models derived from contours. Hydrological Processes, v.14, p.1909-1929, 2000.

Recebido em 23 de julho de 2011 e aprovado em 10 de agosto de 2012 